\title{
Dynamic, multi- and intermodal bike sharing in agent-based modelling
}

\author{
Cornelia Hebenstreit ${ }^{a}$, Martin Fellendorf ${ }^{a}$ \\ ${ }^{a}$ Institute of Highway Engineering and Transport Planning, Graz, University of Technology, Graz, Austria, 8010
}

\begin{abstract}
Negative effects of traffic, like congestion, air and noise pollution are among the reasons why environmentally friendly solutions are promoted. Bike sharing (bs) is intended to strengthen cycling and public transport. Nevertheless, current transport models rarely consider cycling or even bs, in either detail or holistically. In this paper we present an agent based approach to model cycling and in particular bs within the multimodal simulation environment MATSim. Multimodal trips combining public transport and bs are included as well as within day rescheduling of bs trips as agents may not find a bike or empty return space (parking spot). To minimize such cases, choice probabilities were implemented, so that agents only start their bs trip, if sufficient bikes or parking spots are available. The modules presented in this paper were applied using a MATSim model of the city of Vienna. Agent based bs modelling is an inexpensive option to test the impact of a bike sharing system before implementation.
\end{abstract}

Keywords: Bike Sharing; Transport Simulation; MATSim; Route Choice; Agent-based Simulation, Intermodality

\section{Introduction \& Background}

As urbanization is increasing worldwide as is city density, travel speed of motorized vehicles is decreasing in many cities. At present, the average speed of cars in congested cities in London is about $19 \mathrm{~km} / \mathrm{h}$ and in Berlin $24 \mathrm{~km} / \mathrm{h} \mathrm{[1].} \mathrm{At} \mathrm{such}$ low travel speeds bicycle traffic is competitive, especially for short distances. Several cities and private investors implement bike sharing systems (bs) to offer a space efficient and environmentally friendly mobility solution. Bike sharing is a valid option to move quickly within the city, without the need of an own bicycle. Since the private car is still the most comfortable and convenient choice, additional effort has to be undertaken to make the healthy, quiet, energy-efficient and emission-free transport mode cycling more attractive. New options in terms of comfort and scope are now arising with ebikes, also for bs. Two common models exist for bs, station based, where docking stations are required to rent/return the bicycle, and free-floating systems. In the later case bicycles can be taken and returned anywhere within a service area although some public agencies impose parking regulations after bicycles clogged sidewalks and other public spaces. Free-floating bicycles are identified by GPS. Station-based systems (i.e. Vienna, Paris and Lyon) do not offer reservation options. Therefore, modelling of bs differs from the methodology for car sharing [2].

As with other new technical systems, bs will only be successful if the advantages will outweigh. In order to predict the potential usage and success of bs, the system components

(service area, bicycle fleet, station density, station capacity, rental fee, etc.), other transport modes and user behavior must be considered. Mode choice options, city planning and a bicycle friendly environment might further determine if a system will operate successfully. Previous studies have identified travel time reductions if public transport (pt) is used in combination with bs. Nevertheless reductions may not take place at destinations close to pt hubs [3, 4]. In Vienna, Austria, $40 \%$ of bs users are female, and $80 \%$ are younger than 40 years [5]. Also in Australia (Melbourne, Brisbane) bs members are significantly younger but disproportionately male. The distance to the closest docking station is taken as a predictor for membership, and for convenience [6]. In Fargo, North Dakota, key success factors affecting the ridership of bs were analyzed [7] and did not differ greatly to factors influencing bicycle usage in general. Weather, temporal variables (time of day or week) and spatial variables (bicycle infrastructure, land-use...) influence the bs ridership. Influencing factors for cycling were also investigated by other researchers. Safety matters a lot. Four out of five persons are anxious about traffic accidents while riding their bicycle. Street lighting is seen as essential to increase safety $[8,9]$, if it is not area wide available [10]. Other research claimed additional factors which influence route choice and usage of bicycles such as land use, the characteristics of the motorized traffic to be encountered [11, 12], population density, housing or employment, street connectivity $[13,14]$, cycle infrastructure $[15,16]$, pavement conditions $[17,18]$ or a combination of safety, comfort and speed $[15,16]$. In Atlanta, USA, female and senior cyclists are more likely to take longer routes and the deviation from the shortest route depends on the presence of cycle infrastructure,

* Corresponding author. Tel.: +43 3168736723

Fax: +43 316873 4199; E-mail: cornelia.hebenstreit@ tugraz.at

(C) 2019 International Association for Sharing Knowledge and Sustainability.

DOI: $10.5383 /$ JTTM.01.01.002 
bicycle friendly streets, speed limits, confidence and comfort [19]. In Graz, Austria, bicycle travel speed, gender and fitness were identified as key parameters influencing cyclist's route choice [10]. Song et al. [20] present a good summary of cyclist's route choice attributes.

While many criteria for bicycle traffic and bs are already known, cycling is still rarely included in transport models and systematic policy evaluation procedures. Nevertheless the relocation of bs bikes $[21,22,23,24,25]$ and service quality $[26,27,28,29]$, were modelled in detail, which is not the focus of this paper. Numerous multimodal transport models for car traffic and pt exist, but bicycle traffic or bs is mostly sparsely or inaccurately included, and in particular without inclusion of a holistic view of inter- and multimodality. During this ongoing research another group of researchers at TU Berlin developed a multimodal bicycle traffic model in MATSim in parallel. Similar to the approach of this paper, relevant characteristics of infrastructure for cycling were added and the scoring function was updated. With multimodal developments the impact of a bicycle superhighway interacting with other means of transport was analyzed for India [30, 31, 32].

For bs it is important to derive detailed information about the potential of such a system, therefore this paper introduces a module to simulate bs on a fine-grained infrastructure network Within the framework not only the bs system itself is detailed, but also further complex coherences are included. This means criteria such as the transportation network, supply, infrastructure, pt system and schedules, settlement structure (origin-destination relationship), mode choice options and environmental features are considered. The modules presented offer to model bs as single mode as well as a tributary to high ranked pt, within multimodal context.

\section{Methodology}

To simulate bicycles and bike sharing (bs) the open source agent-based simulation software MATSim was enhanced by additional modules. In MATSim, each person (agent) is modelled by individual settings. The actions of agents can cause changes in their environment, which in turn can then cause them to alter their actions. Therefore, there is a feedback loop between agents' decision making and the environment. The relaxed state of the co-evolutionary algorithm is reached if there is no noticeable change in the day plans $[33,34]$. As long as the relaxed state is not reached, iterations are run. An initial day plan of an agent includes activities (e.g. home, work, shopping) and the mode and departure time. Since bad plans will lead to low utility, the remaining plans should level off in utility, as otherwise they will be discarded. Activity chains within a plan stay the same, but activity end times can be shifted together with mode choice. The newly developed bicycle and bs modules provide the simulation of bicycles as well as bike and e-bike sharing. This required the implementation of a station based system. Correlations between choices for bs are given within the module. When bs was chosen as mode, public transport (pt) and walking can also be used, but not vice versa. Some extensions had to be made to the exisiting modelling of bicycle traffic. The new module allows to route and score bicycles according to infrastructure, user group and specifically chosen speed (see 2.1). Furthermore bs was built up from the bottom (see 2.2). For bs single bicycles are modelled so their usage and locations during the whole day can be analyzed. To validate the new module three test cases were used. A real world model of Vienna had been applied for sensitivity and plausibility tests (see 3).

\subsection{Bicycle routing and scoring}

Routing: In order to obtain a realistic spatial distribution, a routing was developed especially for cyclists. Each agent (p) is assigned a user group (u) and a bicycle type (conventional, ebike, road bike). The user group defines how important attitudes $\left(a_{i}\right)$ are for the routing and in further consequence the utility achieved. E.g. it determines how important a safe bike path is for the agent's route choice. In addition, for each agent, a desired cruising speed $\left(v_{p}\right)$ is drawn from normal distribution (derived from measurement data), which depends on the type of bicycle and user group. Furthermore each edge (i) of the bike network receives an evaluation from $0-10$ for all attribute groups (safety, comfort, environment, gradient and other) where 0 indicates best cycle conditions, 10 worst. Further each edge has a length and a maximum permissible infrastructure speed $\left(\mathrm{v}_{\mathrm{i}}\right)$. Every attribute group is multiplied by its user group share $\left(\mathrm{P}_{\mathrm{a}, \mathrm{u}}\right)$, the result is the bike-friendliness $\left(\mathrm{b}_{\mathrm{i}, \mathrm{u}}\right)$. This means every user group has its own value of bike-friendliness attributed for every edge, which gets multiplied by the edge length $\left(l_{i}\right)$ and divided through speed $\left(v_{i, p}\right)$. The result is the personal weighting $\left(\mathrm{w}_{\mathrm{i}, \mathrm{u}, \mathrm{p}}\right)$ for every bike-edge. For speed the minimum of personal $\left(\mathrm{v}_{\mathrm{p}}\right)$ and edge speed $\left(\mathrm{v}_{\mathrm{i}}\right)$ is decisive. The calculation of the personal weighting is introduced in Formula (1) and (2). The Dijkstra-Algorithm uses the personal weighting, to calculate every agent's best route between origin and destination.

$$
\begin{aligned}
& w_{i, u, p}=\frac{l_{i}}{v_{i, p}} * b_{i, u} \\
& b_{i, u}=\sum_{a=1}^{5} a_{i} * P_{a, u}
\end{aligned}
$$

Scoring: Within MATSim utility is used to determine if the equilibrium is reached. In simplified terms, positive utility is gained for performing activities and negative utility is received for travelling. Since most scoring functions already exist, only scoring for travelling by bicycle or bs is explained here. It is necessary to determine how good or poor a detected route is. This depends again on the route, user group and speed. If an agent finds poor conditions in terms of its preferences a lower score will be reached, than for good conditions.

In order to cope with this the perceived travel time $t_{\text {trav,i,perc }}$ was introduced. Our implementation matches to statements of Boekhoudt et al. [35] who introduced the concept of person dependent perception of travel time. In our case, this means that an edge with good cycling conditions, has a lower value than the real travel time, resulting in a higher utility and vice versa. The perceived travel time is calculated by

$$
t_{\text {trav }, i, p e r c}=t_{\text {trav }, i} * \sum_{a=1}^{5} m_{a_{i}} * p_{a, u}
$$

where $m_{a_{i}}$ depends on the characteristics of every travelled edge (see Formula 3 ). This means a route is scored with the help of the perceived travel time, keeping original departure and arrival times for activity scoring. The utility is calculated by summing up the perceived travel times of all edges and multiplying this by the marginal costs of travelling $\left(\beta_{\text {travel }}\right)$. The marginal costs consist of time dependent costs, route determined costs and monetary costs. Monetary costs can be especially important for bs. This means separate utilities for bicycles, bs and e-bs may arise. For a more detailed explanation of the routing and scoring method and general utility formulas in MATSim see [8, 29, 33, 34]. 


\subsection{Station based bike sharing framework}

For the implementation of station based bs, every station is assigned to a network edge and agents must be able to reach this edge by the modes walk and bicycle. Every station has its coordinate, type (e-bike or conventional bike) and contains the number and id of available bicycles and the number of available parking spaces (empty boxes). For e-bikes physical values describing the charging curve need to be provided. The bs module reproduces renting and returning of a bicycle precisely. Interaction activities are included which arise from travelling by pt or bs. Such activities are inserted whenever a mode change between bs and pt is performed. So called legs contain e.g. information about the route and chosen mode from origin to destination. The initial input plan of an agent, is a simple plan, without detailed timing or routing. For bs this plan is transferred to a pre-processed bs plan (Fig. 1).

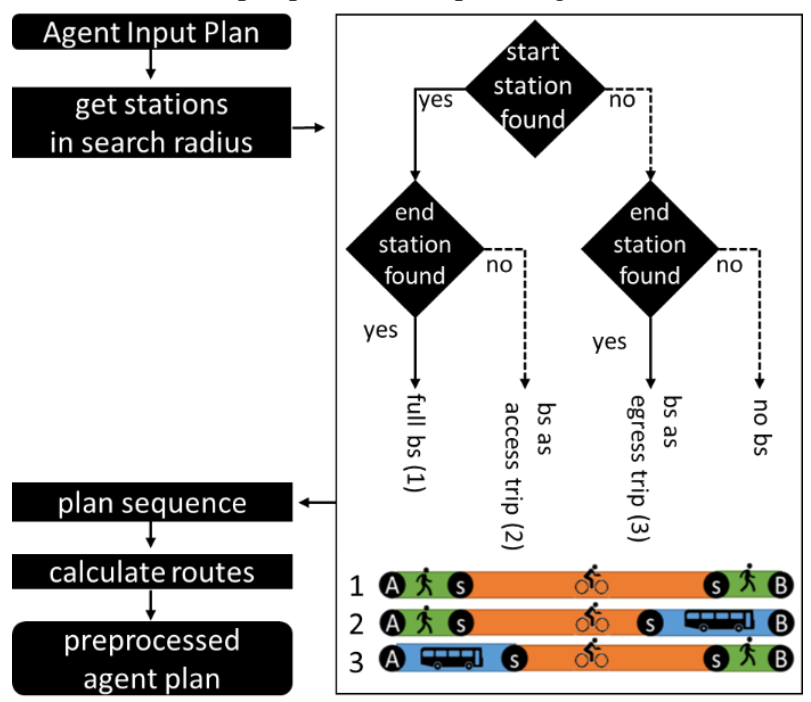

Fig. 1. Methodology to generate a pre-processed bs plan

Bs type, according to station choice: The distances between origin and the nearest station, and between destination and the nearest station are calculated in order to find the start and end station. After this step, the bs type can be derived. It depends on all found stations within the search radius and the beelinetrip-distance from the previous to the next activity. An initial station for departure is found, if there is at least one station within the search radius, which is an input parameter, and can be defined by the modeler. If for both types, the same priority type was found, choice depends on likelihood and on access and egress distance, with advantage for e-bs. If only one start or end station was found bs is combined with pt (access or egress trip). Either start with bs (2) and end the trip with pt, or vice versa (3). Walk trips to and from a station as also the bicycle trip itself are routed. Station choice and routing, firstly result in the best possible bs plan, as at first available bikes or parking spaces are not considered and time reference is not used. Nevertheless, a further constraint can be set by the modeler, since it is possible to set a maximal distance, which will be accepted by the bs agent, for bs standalone use. If this distance is exceeded bs will be combined with pt, as the effort for bicycling would be too high, which is a response to the knowledge that cycling gets rejected whenever a specific trip length is exceeded. As this length depends on local and country circumstances, this length was implemented as an adjustable variable. Bs-pt-exchange-stations, shall only be stations near to high-ranked pt or hubs. Time-reference is added in a second step (see Fig. 2). The agent plan might be adapted whenever an activity ends. The validity of the pre-processed bs plan is thus verified. This means if the agent is about to leave a plan activity i.e. home, the conditional probability gets calculated. There are three options a) the agent keeps to the pre-processed plan, b) the agent still uses bs, but changes the start station and/or end station, due to a better chance of finding bikes/parking or c) the agent changes the mode, because it is unlikely that a bike/parking will be found. Whenever a bs station is reached by an agent, it is checked if bikes (taking), or parking spots (returning) are still available. If not, bs agents can perform choice options (wait, change mode, change station). The process (see Fig. 2) shows, that every step of bs is modelled. Whenever a bs agent ends an activity, this activity is checked if it is of type waiting, bs_interaction, or any other type (yellow). Every time a bs_interaction activity ends, bikes are returned if parking is available for them (violet) or taken if bikes are available (orange). If neither, possible choice strategies (change station/mode, wait) are selected. If any other activity than waiting or bs_interaction ends, the actual plan elements are checked for validity. When the initial station/s still is/are likely to have bikes or parking spots, the agent keeps its plan. If not, the station or mode can be changed (pink). Whenever a bike is taken or returned, the first agent of the list returns or takes a bike (white) and is removed from the waiting list.

\subsubsection{Probability of getting a bike or empty box}

Agents verify their bs trip as they leave their previous planactivity. The agents then search for all stations within their search radius and decide if they start a bs trip, dependent to the found available bikes at the origin station (t) and parking spots at their destination station (r). In this case, the probability of selection, depends on the available bikes or parking spots. The probability that agent $i$ selects an alternative $j$ is given by utility $\mathrm{U}_{\mathrm{ij}}=\mathrm{f}\left(\beta_{\mathrm{j}} \mathrm{x}_{\mathrm{ij}}+\varepsilon_{\mathrm{ij}}\right)$, where $\beta_{\mathrm{j}}$ is the intensity and direction of the influence quantity at alternative $\mathrm{j}$, and $\mathrm{x}_{\mathrm{ij}}$ is the observed influence quantity of alternative $\mathrm{j}$ for all agents $\mathrm{i}$, and $\varepsilon_{\mathrm{ij}}$ is the unobserved error term. Let's assume a station with a defined station size (s) has two alternatives which can be chosen. In the case of taking a bike these two alternatives are taking a bike $\left(t_{y e s}\right)$ or not taking a bike $\left(t_{n o}\right)$, where $t_{y e s}$ consideres the available bikes $\left(\mathrm{n}_{\mathrm{b}}\right)$ of a station and $\mathrm{t}_{\mathrm{no}}$ all empty boxes $\left(\mathrm{n}_{\mathrm{eB}}\right)$, including a control-variable, accordingly $r_{y e s}$ are the available parking spots and $r_{n o}$ the filled boxes. We assume that the utility of each alternative is calculated by

$$
\begin{array}{lll}
\text { Taking: } & U_{t_{y e s}}=n_{b} & U_{t_{n o}}=s \alpha-n_{b} \\
\text { Returning: } & U_{r_{\text {yes }}}=n_{e B} & U_{r_{n o}}=s \gamma-n_{e B}
\end{array}
$$

The utility is calculated with formulas of (4) and (5), the result is shown in Fig. 3. The $\mathrm{x}$ axis states the available objects (yes). The y axis shows the belonging probability, this means how likely it is, that the agent will get a bike/parking at the station, different parameters for $\alpha, \gamma$ can be used. As both probabilities must match that an agent starts a bs trip, $P_{t} \wedge P_{r}$ is valid. Therefore the probability is calculated by $\mathrm{P}_{\mathrm{t}} \mathrm{P}_{\mathrm{r}}$, as both probabilities must be achieved. Let's assume, only one start and end station was found within search radius. The start station has 25 bike boxes in total and 3 available bikes, the end station has 30 boxes and 5 empty boxes. With $\alpha$ is $\gamma$ is 0.25 , $\mathrm{P}_{\mathrm{t}}$ is $45 \%$ and $\mathrm{P}_{\mathrm{r}}$ is $95 \%$, which yields $\mathrm{Pt} \wedge \mathrm{Pr}$ is $43 \%$. This means the agent has a $43 \%$ chance to start the bs trip. If there is more than one start or end station within search radius, the ones with the highest probabilities are taken into account. 
TIME DEPENDENT PLAN ADAPTION/EXECUTION:

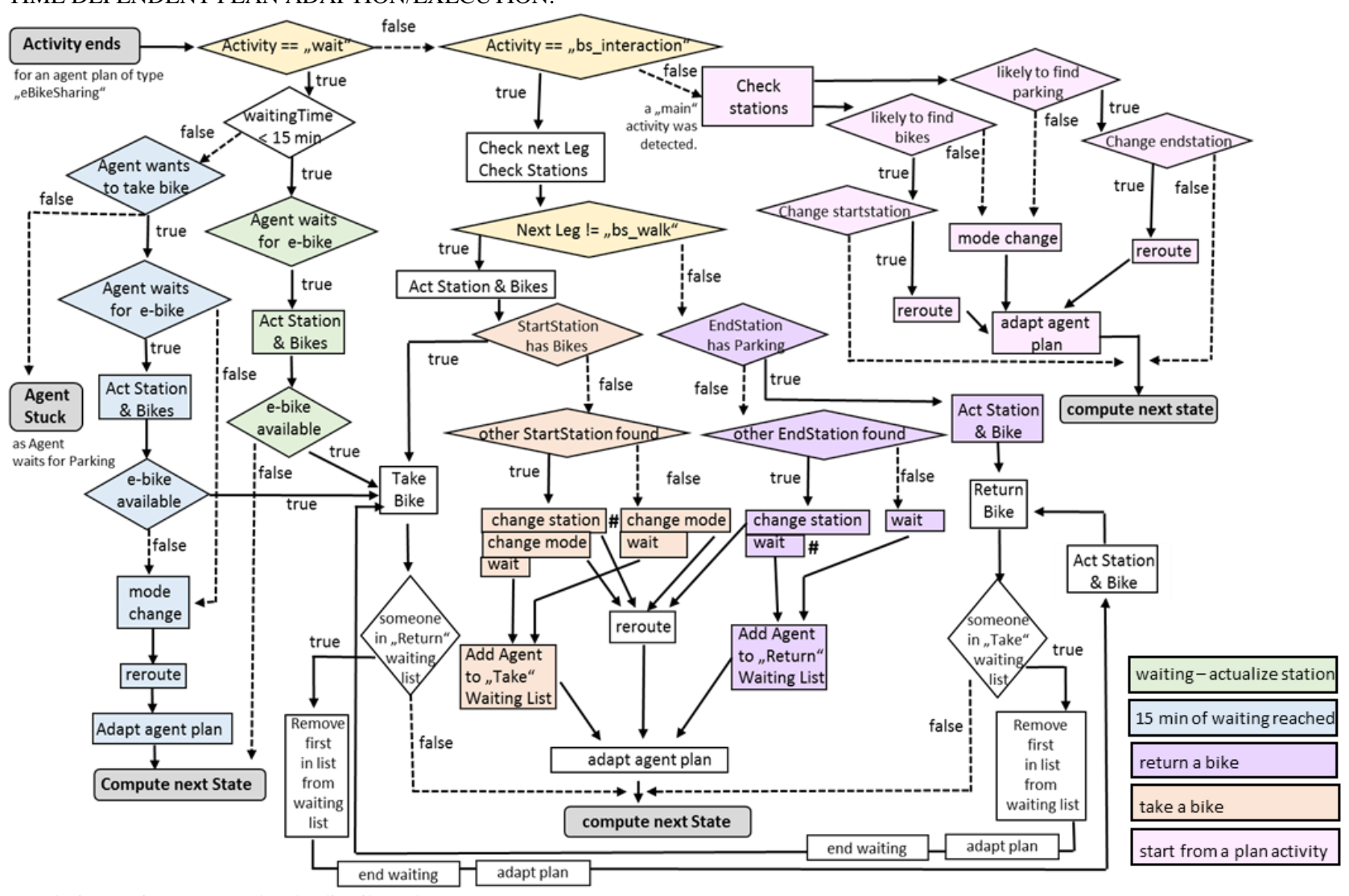

\# Choice options are randomly distributed.

The highest likelihood is given to, where the highest utility is expected. Choices of modes depend on distance.

Fig. 2. Flow chart of the time-dependent processes of the bs module. The pre-processed agent-plan is verified and possibly adapted.

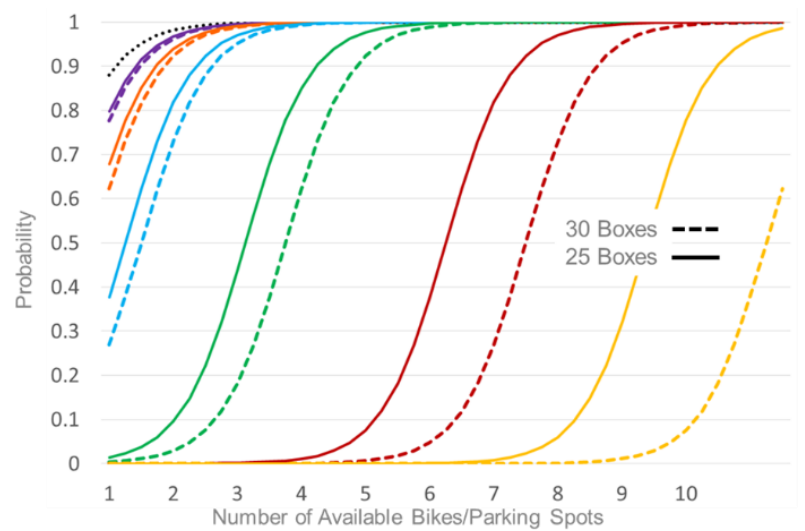

Value for $\alpha$ or $y---0.025---0.05---0.1 \quad---0.25 \quad---0.5 \quad--0.75 \quad \cdots \cdots .0$

Fig. 3. Results of the probability of selection, considering different total numbers of bike boxes and $\alpha=\gamma$ values

$$
P_{i j}=\frac{e^{U_{i j}}}{\sum_{j}^{n} e^{U_{i j}}} \quad P_{t}=\frac{e^{U_{t y e s}}}{e^{U_{\text {tyes }}}+e^{U_{t_{n o}}}}
$$

\subsubsection{Station and bike update}

A bs station is updated whenever a bike is taken or returned, or if the activity waiting for an e-bike ends. Conventional bikes and e-bikes are treated differently, because of the battery which is essential for an e-bike, so e-bikes get updated every time an e-bike is taken. All e-bikes, which are situated at the station in focus are updated. Only bikes which are at least $10 \%$ charged can be taken for travel purposes by the agents. Agents always take the e-bike with the highest state of charge. Whenever an ebike is returned, its charge status is updated, as it was discharged. If a minus value is detected, the agent who returned the bike will obtain additional travel time, which results in a higher negative utility for travelling, but certainly recharging never starts sub-zero.

\subsubsection{Charging and discharging}

E-bikes have a charging and discharging energy flow. A charging curve was deposited, which takes the quantities from the input file. Every single bike is modelled as an own object. This means for e-bikes the quantities for charging must be stored in the input file. Every single bike is modelled as an own object, and may have own battery attributes. This means it is possible to simulated different battery types as well.

For the discharging process, a simplified linear loss was assumed. In reality discharging is dependent on factors such as battery support, gradients, travel speed, weight of the user, head wind and others, however, we only take the average scope into account, which can be set as input value.

Formula (6) was implemented for charging, which represents a UIa charging curve for Lithium-Ion batteries. Ebery bike is an own object, as a result the location of bs bikes, the daily load factor and usage, the kilometers travelled per day and bike, etc. are possible to be analyzed.

$$
\text { SOC }=\frac{\mathrm{q}(\mathrm{t})}{\mathrm{Q}} \quad \text { or } \quad S O C=1-\mathrm{e}^{-\frac{\mathrm{t}}{\mathrm{R} * \mathrm{C}}}
$$




\subsubsection{Choice options}

Let's assume the agent uses bs, but when he arrives at the station neither a bike/parking spot was found. If so, agents can choose different choice strategies. There are three choices available, change station, change mode or wait. The type is chosen randomly, with respect to certain boundary conditions (i.e. waiting queue, trip length). Programming becomes more complex if pt was also used by the agent in the course of a trip. Some pre-processing steps are thus necessary. For subsequent iterations, it must be ensured, that all bs interactions or waiting activities will not be used as activities to which an agent wishes to travel to, whenever a mode change is prevalent.

\subsubsection{Waiting}

If waiting was chosen, the agent is added to the stations waiting list. Here the first in first out principle was applied, but never more than 2 agents will simultaneously wait at one station. Waiting is implemented as five consecutive activities of 3 minutes of waiting and these are added as activities after the actual activity. If the entire waiting time $(15 \mathrm{~min})$ has been spent fruitlessly, waiting is aborted. For an agent waiting to take a bike, a mode change is conducted. This course of action is the same for returning a bike, but if no bike was taken during the latency time, mode choice is not an available option. The agent could not return his bike and will not achieve good utility for the plan. This is depicted by a very slow walk to the next activity. If, during plan handling, waiting was found and the waiting time is less than $15 \mathrm{~min}$, the e-bike station of this agent gets updated, because if an unloaded e-bike was present, this bike might meanwhile be sufficiently charged. If the maximum waiting time was reached an agent waiting to return a bike, will abort waiting, resulting in a low utility. For an agent waiting for a bike, if a bike/e-bike is still not available a mode change will be conducted.

\subsubsection{Relocation}

As relocation might decide if a bs system will work, a framework for relocation was implemented. The used methodology is simple and allows to relocate bicycles in a given time interval. Nevertheless no real relocation traffic is generated. Within the given interval either the most saturated or the emptiest station is crucial for the start of the process. The simple logic exchanges bicycles from the emptiest and to the fullest stations or vice versa, only considering beeline distance for a time offset, but no network load. This means between collecting the bicycles and backfilling them a time offset is considered. The relocation logic was not the key of this work. However, the method of relocation has been used as satisfactory heuristic.

\subsection{Validation of the bike sharing functionalities}

The module was fault checked, refined and tested on plausibility, using validation examples. One example with only 3 stations, but a detailed network description focused on the routing and taking, returning and charging process review. This means stations, bikes and the routing algorithm were proved. Another example with 5 stations was constructed to examine those cases where agents do not find bikes or parking spots and are then first added and later on removed from the waiting list. The waiting list handling was checked with this example. The third validation example had $17 \mathrm{bs}$ stations and a greater catchment area. It was used to prove the station selection and derivation of bs types (if bs is used combined with pt or not). Further to this plan resetting was also tested. All examples were modelled multi and intermodal including bike, walk, cars and pt. These examples were all used to examine adaption within the day plan. Since agent decisions to change their plans during the ongoing simulation depend on interaction with other agents, time dependency and changes within agent plans was an issue we examined very closely.

As free-floating bs has also recently been introduced with increasing frequency in cities, it was implemented too. For free floating bs, bikes can normally be reserved beforehand. In our tool this happens once the agent leaves a plan activity. The nearest bike is taken, if it is within the search radius. If not a pt trip gets conducted. As this process is simpler than station based bs, it is not described here in detail.

\section{Real world application, Vienna}

Both the bicycle module and the bike sharing module were applied for a model of the City of Vienna. Therefore a base case, with the means of transport car, public transport (pt), walk and bicycle was developed and calibrated. The aim of the model was to perform measure sensitivity tests for both developed modules. In this section results of three sensitivity tests of the Vienna model are shown. The first test shows the routing behavior results, the second test shows the sensitivity of both probability and relocation effects, and the third test shows the mode choice sensitivity.

\subsection{Routing results}

Three different user groups were defined for the model. One with high safety needs, one with equally important attributes and one with low safety need. Attribute categories were not implemented in a high level of detail, this means an estimation was done only dependent on the bicycle infrastructure type. For the Vienna model no slope was used. This means the algorithm explained in section 2.1 also works if just little data is available. If no data is available, the routing would deliver the shortest path in terms of travel time.

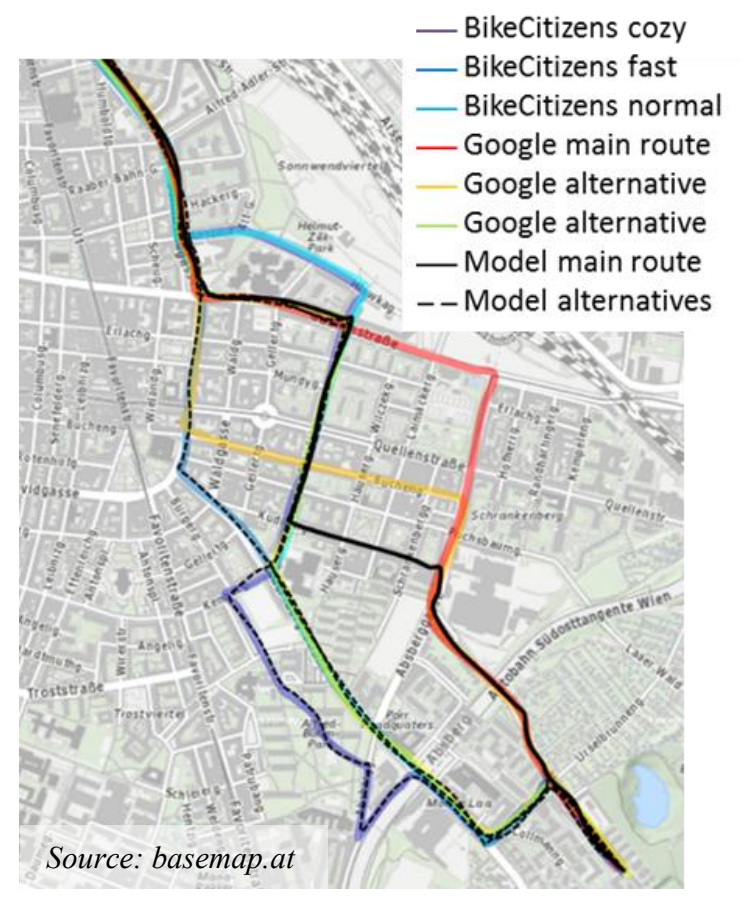

Fig. 4. Routing results of BikeCitizens, Google and the model 
Figure 4 shows results of different routing tools, compared to the model output. BikeCitizens an App, developed for bicycle routing and tracking, allows to route with cozy, fast and normal cycling behavior, and stated four routes. The wellknown Google routing, states one main route (red) and two routing alternatives. The model output presents four options, the drawn through line represents the highest usage. As it can be seen similar routes arise, but divergence exists between all routings.

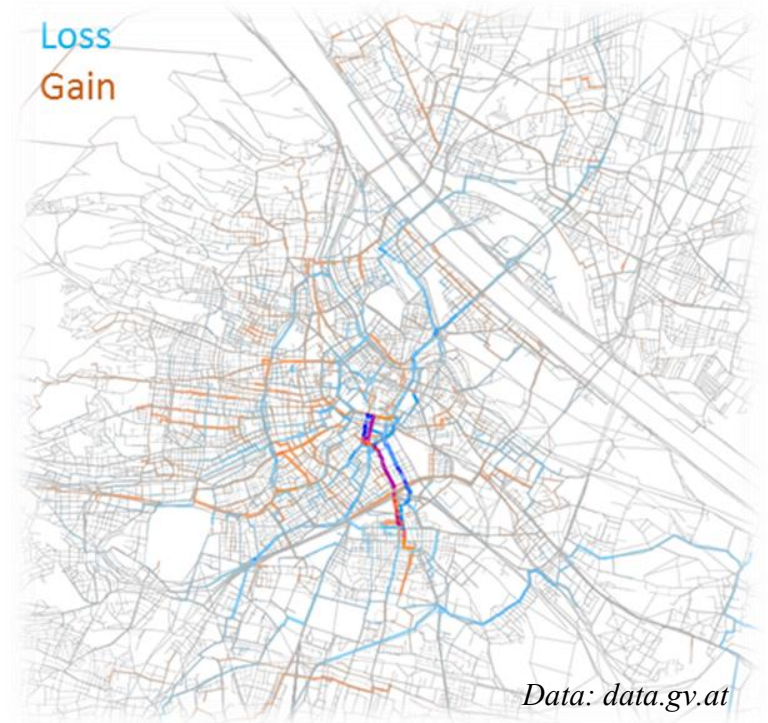

Fig. 5. Gains and losses due to the fast cycle connection in the south of Vienna.

Additionally a use case was simulated and analyzed, where fast cycle connections were opened in the model. With this example, the losses and gains within the network could be detected. Interestingly having a fast cycle connection brings gains especially very close the connection but the effects reach way further. Figure 5 shows gains and losses of the south direction.

\subsection{Effects of the relocation interval and likelihood of starting a bike sharing-trip}

With a single iteration where all agents used bs, both the relocation interval ( $\mathrm{R}$, in seconds) and likelihood of finding a bicycle/empty box (P) could be analyzed. Therefore ten simulation runs were performed, each of them had different values of $R$ and $P$.

Figure 6 shows the results of the percentage of taking and returning actions, in respect to the initial iteration. The initial iteration has no chronological time handling, therefore station size and load is not considered. This means the initial iteration states the maximal possible bike sharing trips and only considers stations and search radius.

The left side of Figure 6 shows the effect of P. The lower the number of bicycles and boxes must be for agents to start their bike sharing trip, the more agents use bike sharing. Nevertheless the lower this number is, the more unfruitful interaction (no bicycle, no empty box) are generated as well.

The denied interactions can be seen on the right side of the figure. It could be proved that a shorter relocation interval brings possible benefits for the bs-system if there is a high utilization. This means with a shorter interval both, more fruitful interactions can be performed and less impossible interactions were achieved (see e.g. arrows in Figure 6).

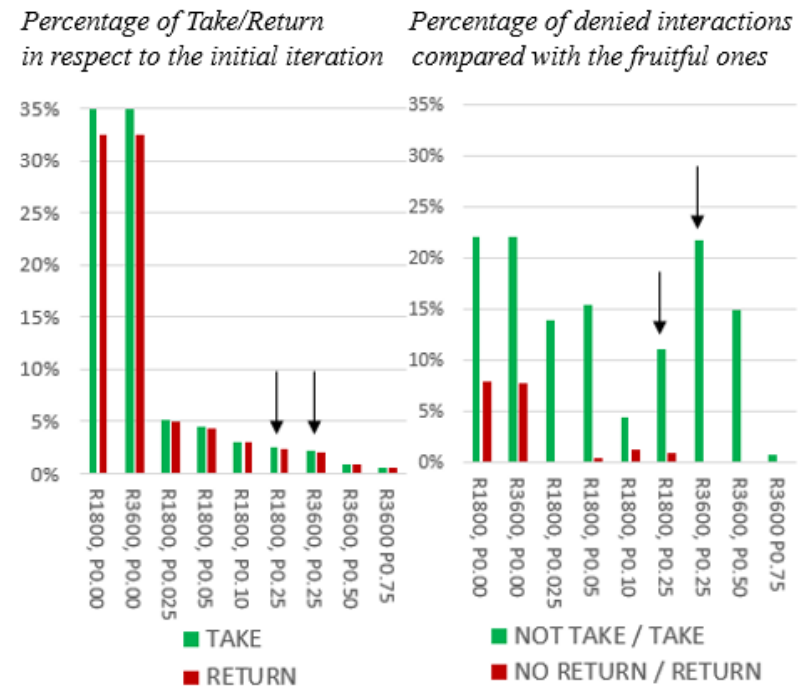

Fig. 6. Bs interaction results, of full bs iteration only

\subsection{Mode choice sensitivity}

Using different simulation settings, measure sensitivity tests and the effects on bs were verified and analyzed. To compare different effects, $\mathrm{P}$ was set to 0.05 , and $\mathrm{R}$ to 1800 for all subsequently simulated cases. For the simulation result shown here only a $1 \%$ demand sample was used, and public transport was not routed. For the analysis of the relaxed state five cases were simulated:

a) Base case: The disutility for travelling was retained of the base simulation, representing the Vienna modal split.

b) Doubled pt disutility case: Disutility for public transport (pt) of the base case was doubled.

c) Doubled car disutility case: Disutility for car traffic of the base case was doubled.

d) E-bike sharing: Same disutility values as the base case, but using e-bike sharing instead of conventional bs.

e) Additional 10 stations: New stations were implemented in the northern districts $\left(21^{\text {st }}\right.$ and $\left.22^{\text {nd }}\right)$ of Vienna.

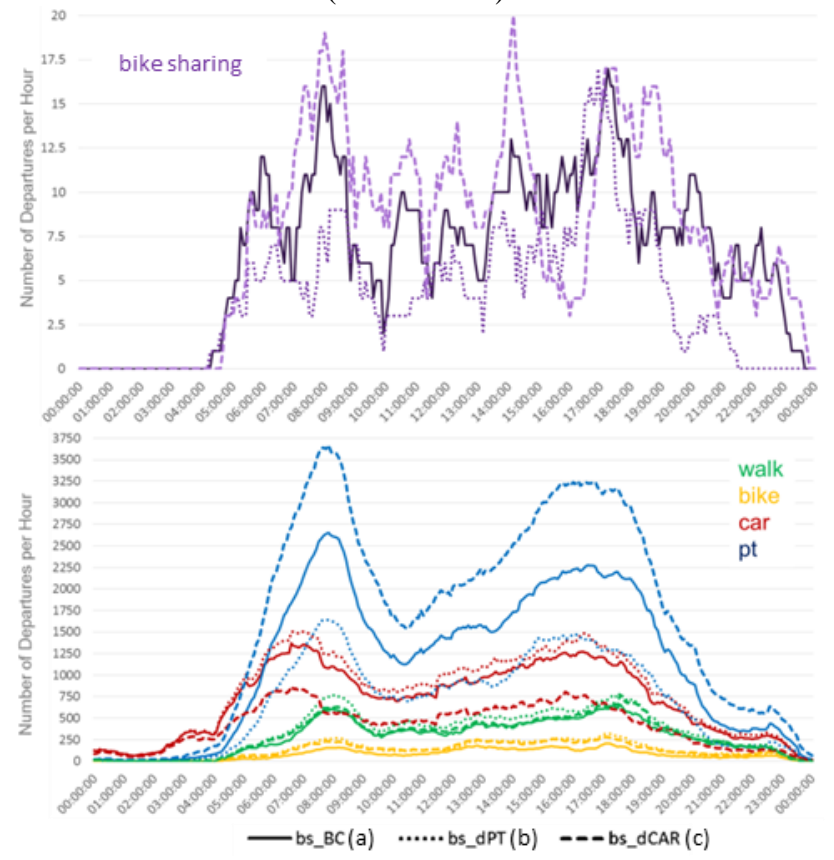

Fig. 6. Hydrograph of departures for the cases a), b), c) of the Vienna model, using a $1 \%$ sample 
As expected, in b) pt usage and in c) car usage declines compared to a). Further as a doubled pt disutility in b) has effects on bs-pt interaction too, bs usage is highest in c), which can be seen in Figure 6. The hydrograph shows departures for every means of transport. This means if more than one public transport line was used more than one departure is present.

The effects of newly implemented stations e) lead to a general usage gain, so does the transformation of conventional bike sharing to e-bikes d), as e-bikes offer more comfort and a higher riding speed.

In values this means that compared to the base case, bike sharing usage increased by $42 \%, 23 \%$ and $17 \%$ for e), d) and c) respectively. For the doubled pt disutility b) bike sharing usage declined by $37 \%$. Subsequently some results for the additional 10 stations (e) are shown.

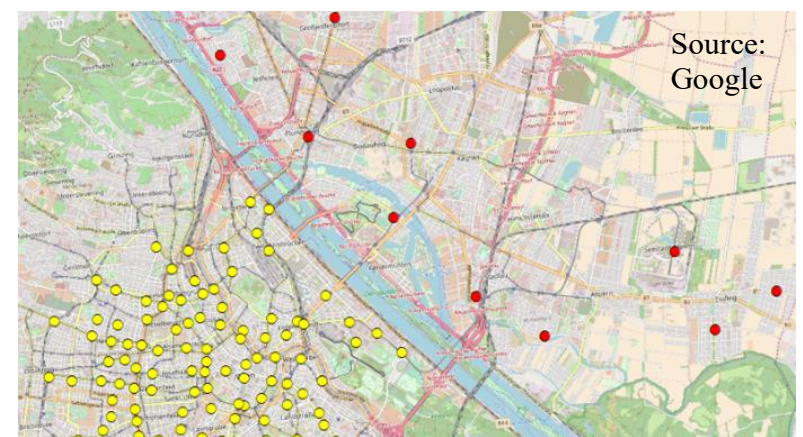

Fig. 7. Map of the original stations of bike sharing in Vienna (yellow, not all shown) and additional 10 stations (red)

For the new stations (e), as shown in red in Figure 7, a positive effect of bike sharing was achieved (see Figure 8). This means more stations brought more bike sharing usage, a very slight increase in public transport (pt) and walk trips, and a reduction in car trips. Therefore it is important to implement bike sharing intermodal. This means at least in the transport model a benefit of new station, even if the locations of the stations are not densely distributed, was found.

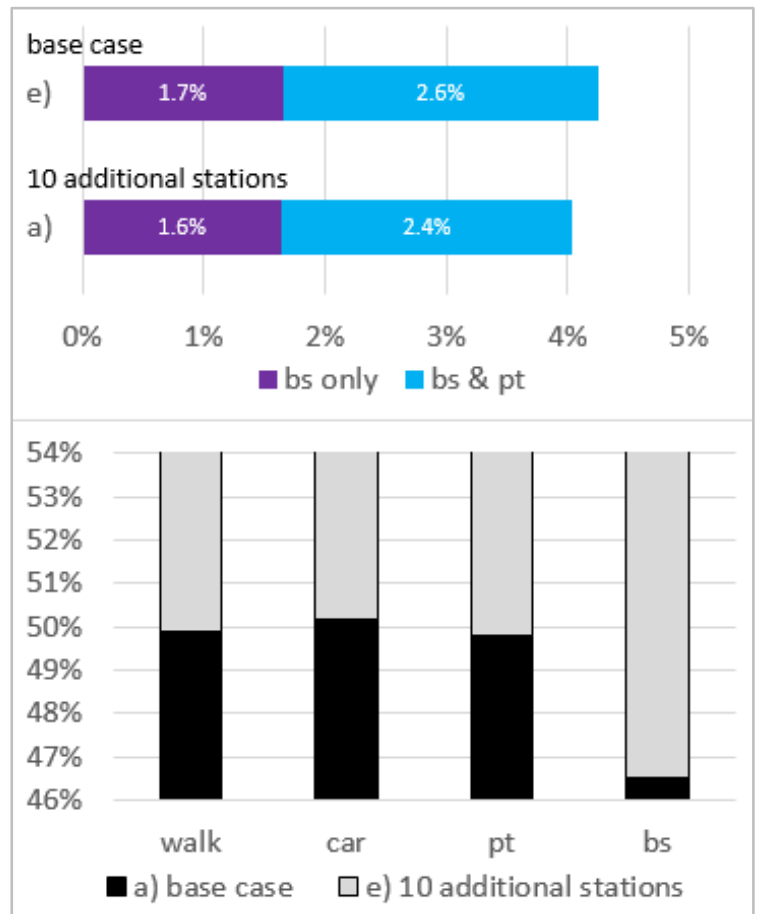

Fig. 8. Share of means of transport for the base case (a) and the additional ten stations case (e),

\section{Conclusion}

The work of this paper introduced two new MATSim modules which allow to simulate bicycle traffic, and bike sharing in combination with other means of transport. The bike sharing module allows agents to perform intermodal trips or multimodal dayplans. In their daily travel plans the travel modes walking, bike sharing and public transport can be combined and mixed up. Several enhancements were made to extend MATSim so that bs can be tested within a multimodal and intermodal environment.

The methodology of the implementation, measure sensitivity and hypothesis tests is described within scope of the work presented in this paper. Further bs and e-bs were applied for a simulation model of the City of Vienna, Austria. In Vienna a conventional station based bike sharing system, as implemented in the base case, exists.

From the present position and outlook the results of the simulated cases, and hypothesis, and measure sensitivity tests that are being carried out appear to be very promising. The bike sharing module was developed to simulate bike sharing in a very high level of detail for gaining very precise. With those two modules holistic models can be developed. This detailed depiction brings numerous advantages, the downside is the computation effort, as large scale applications are only operational at very high computation costs.

Since parameters for routing and utility calculation were only surveyed in a pretest, with focus on the implementation and not on the simulation results [10], parameter estimation is necessary further work. In this paper it was not the idea to focus on parametric rating, since up to now the module design, implementation, computation and sensitivity tests had priority. This means parametric fitting would be a further step, and one which will depend on specific personal but also locationrelated behavior.

However, the current implementation allows already several scenarios of practical relevance such as changes in modal split due to bs and bs as tributary to strengthen public transport, can be tested. It would also be possible to calculate occupancy rates of bicycles and load factors. By testing these scenarios a lack of suitable bs stations or not well used ones can also be identified.

\section{Acknowledgments}

Research presented here is partly supported by the research projects MatchSim and (R)adOmnes, both financed by the Austrian Ministry for Transport, Innovation and Technology (BMVIT) within the national funding program "Mobilität der Zukunft". Their support is greatly acknowledged.

\section{References}

[1] Forbes Magazine; 2008; Europe's Most Congested Cities

[2] Ciari F., 2012; Sharing as a key to rethink urban mobility: investigating and modelling innovative transport systems, dissertation, ETH Zurich.

[3] Jäppinen, S., Toivonen, T., Solonen M. (2013), Modelling the potential effect of shared bicycles on public transport travel times in Greater Helsinki: An open data approach. $\begin{array}{llll}\text { Applied Geography } 43 . & 2013 . & \text { 13-24. }\end{array}$ https://doi.org/10.1016/j.apgeog.2013.05.010

[4] Klingler, T. (2017), Moving from monomodality to multimodality? Changes in mode choice of new residents. 
Transportation Research Part A. https://doi.org/10.1016/j.tra.2017.01.008

[5] Schneeweiß, H. (2012), Das Fahrradverleihsystem Citybike Wien: Motive, Charakteristika und Perspektiven der Nutzung, Diplomarbeit.

[6] Fishman, E., Washington, S., Haworth, N., Watson A. (2015), Factors influencing bike share membership: An analysis of Melbourne and Brisbane. Transportation $\begin{array}{llll}\text { Research Part A } 71 . & 2015 . & 17-30 .\end{array}$ https://doi.org/10.1016/j.tra.2014.10.021

[7] Mattson, J., Godavarthy R. (2017), Bike share in Fargo, North Dakota: Keys to success and factors affecting ridership. Sustainable Cities a. Society 34. 174-182. https://doi.org/10.1016/j.scs.2017.07.001

[8] Sener, I., Eluru, N., Bhat, C. (2009), Who are Bicyclists? Why and how much are they bicycling? TRR 2134. 63-72. https://doi.org/10.3141/2134-08

[9] Menghini, G., Carrasc,o N., Schüssler, N., Axhausen K.W. (2010), Route choice of cyclists in Zurich. Transportation $\begin{array}{llll}\text { Research } & \text { Part } & \text { A. } & \text { Vol }\end{array}$ https://doi.org/10.1016/j.tra.2010.07.008

[10] Hebenstreit, C., Fellendorf M. (2017), Multi- and intermodal Trip Chain Simulation for individual daily Routines, using Bicycles. Proceedings for 7th Transport Research Arena TRA 2018.

[11] Segadilha, Sanches, (2014), Identification of factors that influence Cyclists' route choice. Social and Behavioral $\begin{array}{llll}\text { Sciences. } & \text { Vol } & 60 . & 372-380 .\end{array}$ https://doi.org/10.1016/j.sbspro.2014.12.149

[12] Parkin, J., Wardman, M., Page, M. (2008), Estimation of the determinants of bicycle mode share for the journey to work using census data. Transportation 35(1). 93-109. ISSN 1572-9435. https://doi.org/10.1007/s11116-0079137-5

[13] Saelens, B.E., Sallis, J.F., Frank, L.D. (2003), Environmental correlates of walking and cycling: findings from the transportation, urban design, and planning literatures. Annals of Behavioral Medicine 25. Issue 2. 8091. https://doi.org/10.1207/S15324796ABM2502_03

[14] Crane, R. (2000), The influence of urban form on travel: an interpretive review. Journal of Planning Literature 15(1). 3-23. https://doi.org/10.1177/08854120022092890

[15] Broach, J., Dill, J., Geliebe J. (2012), Where do cyclists ride? A route choice model developed with revealed preference GPS data. Transportation Research Part A. n46. 1730-1740. https://doi.org/10.1016/j.tra.2012.07.005

[16] Snizek, B., Nielsen T.A.S., Skov-Petersen H. (2013) Mapping bicyclist's experiences in Copenhagen. Journal of Transport Geography. Vol 30. https://doi.org/10.1016/j.jtrangeo.2013.02.001

[17] Landis, B., Vattikuti, V., Brannick M. (1997), Real-time human perceptions toward a bicycle level of service. Transportation Research Record. n1578, 119-126. https://doi.org/10.3141/1578-15

[18] Stinson, M., Bhat C. (2004), Frequency of Bicycle Commuting: Internet-Based Survey Analysis. Transportation Research Record. n1878. https://doi.org/10.3141/1878-15
[19] Misra, A., Watkins, K. (2017), Modeling Cyclists Willingness to Deviate from Shortest Path Using Revealed Preference Data; Transportation Research Board, 2017 Annual Meeting. 17-03556.

[20] Song, Ni, Li (2017), Understanding cyclists“ risky route choice behavior on urban road sections. Transportation Research https://doi.org/10.1016/j.trpro.2017.05.356

[21] Pal, A., Zhang, Y. (2017), Free-floating bs: Solving reallife large-scale static rebalancing problems; Transportation Research Part C: Emerging technologies. $\begin{array}{llll}\text { Vol } & 80 . & 2017 . & 92-116 .\end{array}$ https://doi.org/10.1016/j.trc.2017.03.016

[22] Forma, I.A., Raviv, R., Tzur M. (2015), A 3-step math heuristic for the static repositioning problem in bikesharing systems. Transportation Research Part B: Methodological. Vol 71. Issue C. 230-247. https://doi.org/10.1016/j.trb.2014.10.003

[23] Erdogan, G., Battarra, M., Wolfler Calvo R. (2015), An exact algorithm for the static rebalancing problem arising in bicycle sharing systems. European Journal of Operational Research. V245-3. 667-679. https://doi.org/10.1016/j.ejor.2015.03.043

[24] Abdelmoumene Kadri, A., Kacem, I., Labedi K. (2016), A branch-and-bound algorithm for solving the static rebalancing problem in bicycle-sharing systems. Computers \& Industrial Engineering. Vol 95. 41-52. https://doi.org/10.1016/j.cie.2016.02.002

[25] Faghih-Imani, A., Hampshire, R., Marla, L., Eluru, N. (2017), An empirical analysis of bs usage and rebalancing: Evidence from Barcelona and Seville. Transportation Research Part A: Policy and Practice. Vol 91, 177-191. https://doi.org/10.1016/j.tra.2016.12.007

[26] Cruz, F., Subramanian, A., Bruck, B.P., Iori, M. (2017), A heuristic algorithm for a single vehicle static bs rebalancing problem. Computers \& Operations Research. Vol 79. 19-33. https://doi.org/10.1016/j.cor.2016.09.025

[27] Frade, I., Ribeiro, A. (2015), Bike-sharing stations: A maximal covering location approach. Transportation Research Part A: Policy and Practice. https://doi.org/10.1016/j.tra.2015.09.014

[28] Alvarez-Valdes, R., Belenguer, J.M., Benavent, E., Bermudes, J.D., Vercher, E., Verdejo, F. (2016), Optimizing the level of service quality of a bike-sharing $\begin{array}{llll}\text { system. Omega. } & \text { Vol } 61 . & 163-175 .\end{array}$ https://doi.org/10.1016/j.omega.2015.09.007

[29] Romero, J.P., Ibeas, A., Moura, J.L., Benavente, J., Alonso, B. (2012), A simulation-optimization approach to design efficient systems of bike-sharing. 15th meeting of EURO Working Group on Transportation. EWGT 2012. Procedia - Social and Behavioral Sciences. Vol 54. 646665. https://doi.org/10.1016/j.sbspro.2012.09.782

[30] Ziemke, D., Metzler S., Nagel K. (2017), Modelling bicycle traffic in an agent-based transport simulation, Procedia Computer Science. Vol 109. https://doi.org/10.1016/j.procs.2017.05.424

[31] Agarwal, A., Ziemke, D., Nagel, K. (2018), Bicycle Superhighway: An Environmentally Sustainable Policy for Urban Transport. VSP Working Paper 17-16, TU Berlin. 
[32] Ziemke, D., Metzler S., Nagel K. (2018), Bicycle traffic and its interaction with motorized traffic in an agent-based transport simulation framework, Future Generation Computer Systems. https://doi.org/10.1016/j.future.2018.11.005

[33] Balmer, M., Rieser, M., Meister, K., Charypar, D., Lefebvre, N., Nagel K. (2009), Matsim-T: Architecture and Simulation Times. Multi-Agent Systems for Traffic and Transportation Engineering. 57-78. https://doi.org/10.4018/978-1-60566-226-8.ch003

[34] Raney, B. (2005), Learning Framework for Large-Scale Multi-Agent Simulations. Dissertation. ETH Zurich. Switzerland.

[35] Boekhoudt, C., Te Brömmelstroet, M., \& Thrüsh, M., (2017), Mechanismes achter routekeuzes fietser, tijdperceptie en state of mind. In: Verkeerskunde 05-17 p. 14-1 